PACS: 42.25.-P, 42.25.-Hz, 42.87.-Bg

\title{
Quadric hologram-based self-conjugation of vortex beams
}

\author{
P.V. Polyanskii, Ch.V. Felde \\ Chernivtsi National University, 2, Kotsyubinsky str., 58012 Chernivtsi, Ukraine \\ Phone/fax: +3803722 44730 \\ E-mail: polyanskii@itf.cv.ukrtel.net
}

\begin{abstract}
Self-conjugation of singular (vortex) beams by a static nonlinearly recorded hologram is reported. Diagnostics of phase-conjugated vortices is implemented using an original technique based on Young's model of diffraction phenomena. It has been shown that the phase-conjugated vortex is characterized by the same topological charge as the vortex reversed through four-wave degenerate mixing being different in sign from the vortex reflected by a common mirror.
\end{abstract}

Keywords: singular optics, optical vortices, optical phase conjugation, quadric hologram, edge diffraction wave, Young's interference experiment.

Manuscript received 24.11.04; accepted for publication 18.05.05.

\section{Introduction}

Until very recently, a phase conjugation (PC) of optical beams supporting isolated vortices [1] was implemented by the four-wave degenerate mixing (FWDM) technique alone using photorefractive crystals [2]. A few papers have been devoted to this problem [3, 4]. Meanwhile, PC of optical vortices may be applied for solving widely known scientific and practical problems such as a study of topological reactions, mutual transformations of singular beams of various kinds, PC-interferometry, optical traps and tweezers with multiply-charged optical vortices, laser gyro, etc. In pioneer papers [3, 4], optical vortices were produced by the computer-generated hologram (CGH) technique [5], and the direction of twirling of a phase helicoids was determined interferentionally $[6,7]$, namely, by the direction of typical bifurcation of an interference fringe observed in case of a coherent superposition of an off-axis reference wave and the tested singular beam. The important result of the studies $[3,4]$ consists in the direct experimental confirmation of the difference of the phase-conjugated optical vortex and one resulting from a common mirror reflection in their signs of topological charges.

In the context of our study, it is noteworthy that the interest to phase singularities in optical fields was initially stimulated just by the investigations of optical PC through stimulated Brillouin scattering (SBS) [6], being closely related with the searching for the factors limiting the quality of self-conjugation of a wave front. The study [6] dealt with the developed speckle fields obeying the Gaussian statistics and supporting a lot of vortices (namely, one vortex per one speckle). At the same time, for isolated optical vortices with relatively simple wave front (kind of doughnut Laguerre-Gaussian modes [1]) SBS-PC is impracticable due to violating the Gaussian statistics of the field or, in other words, due to insufficient spatial inhomogeneity of a wave being the subject of PC.

In this paper, we report a PC of vortex optical beams using a static hologram nonlinearly recorded with a standing reference wave [8-10]. Initially, this technique was developed to implement a holographic associative memory with a true brightness tone rendering using the signal beams with a developed speckle structure. However, in contrast to SBS-PC (and like to FWDMPC), wave front self-conjugation using a static nonlinearly recorded hologram proves to be applied to the fields of arbitrary complexity, including plane, spherical, diffraction waves, etc. [11]. It was this circumstance that became the main prerequisite for applying this technique to PC of optical beams supporting isolated vortices.

\section{The principle of a quadric hologram-based wave front self-conjugation}

The linear theory of holography is based on a supposition of proportionality of an amplitude response of the hologram, $T_{a}(\mathbf{r})$, at each point with the position vector $\mathbf{r}$, to the exposure $E(\mathbf{r})=t I(\mathbf{r})$, where $t$ is an exposure time, and $I(\mathbf{r})$ is an intensity distribution resulting from interference between the signal and reference waves. In general, however, holographic recording is not linear in the known sense, and the hologram amplitude response can be expanded into the power series expansion

$T_{a}(\mathbf{r})=\sum_{l=0}^{n} T_{l}[I(\mathbf{r})]^{l}$, 


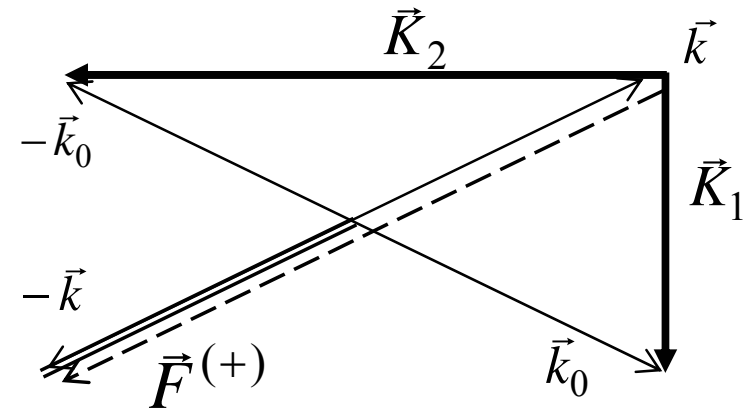

Fig. 1. Vector diagram illustrating a QH-based PC. The total grating with the grating vector $\mathbf{F}^{(+)}$provides re-scattering $\mathbf{k} \rightarrow-\mathbf{k}$.

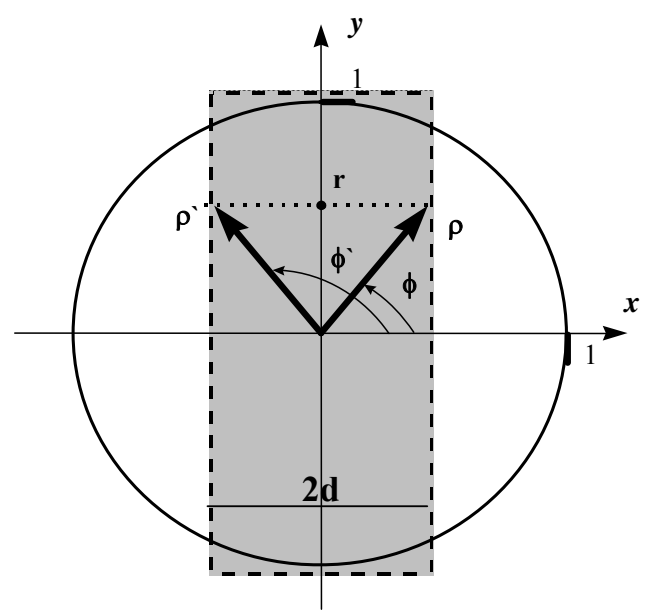

Fig. 2. Notations for analysis of the Young strip interference experiment or testing the vortex beams: $d-$ a halfwidth of the strip, $\boldsymbol{\rho}=(\rho, \varphi)$ and $\boldsymbol{\rho}^{\prime}=\left(\rho^{\prime}, \varphi^{\prime}\right)-$ the position vectors of the edge retransmitters involved into formation of an interference pattern at the height $r$.

where $T_{l}=c_{l} t^{l}$, and $c_{l}=(l !)^{-1}\left[\partial^{l} T_{a}(\mathbf{r}) / \partial E^{l}(\mathbf{r})\right]$. Practical conditions of validity of the representation (1) are sufficiently common. There is the possibility to choose the signal-to-reference amplitude ratio approaching to unity as well as exposure and development duration considerably exceeding those for providing "linear holographic recording”. Besides, a phase holographic recording is non-linear by definition. Nonlinearity of holographic recording manifests itself, first of all, in appearance of the higher diffraction orders. Keeping the term of the representation (1) with $l=2$ constitutes the quadric hologram $(\mathrm{QH})$ approximation $[9,10]$ (or, in terms of [12], "a second-order hologram, i.e., a hologram between two holograms").

Let us consider a hologram of the signal beam, $G(\mathbf{r})=A \exp [i(\omega t-\mathbf{k r}+\varphi)]$, nonlinearly recorded with a standing reference wave, constituting of two oppositely directed beams,

$$
\begin{aligned}
& \Omega(\mathbf{r})=\Omega_{1}(\mathbf{r})+\Omega_{2}(\mathbf{r})=a \exp \left[i\left(\omega t-\mathbf{k}_{\mathbf{0}} \mathbf{r}+\varphi_{1}\right)\right]+ \\
& +b \exp \left[i\left(\omega t+\mathbf{k}_{\mathbf{0}} \mathbf{r}+\varphi_{2}\right)\right] .
\end{aligned}
$$

The complex amplitude of the signal LaguerreGaussian beam is determined by the well-known representation [1]; the explicit form of this representation is inessential for the purpose of this study. An amplitude response of such QH has the form

$T_{a}(\mathbf{r})=\sum_{l=0}^{2} T_{l}\left[\mid G(\mathbf{r})+\Omega_{1}(\mathbf{r})+\Omega_{2}(\mathbf{r})^{2}\right]{ }^{l}$.

The quadratic component of the representation (2) includes, among others, the term $\left\{2 T_{2} \Omega_{1}(\mathbf{r}) \Omega_{2}(\mathbf{r})\left(G^{*}(\mathbf{r})\right)^{2}\right\}$. It is this term that is responsible for $\mathrm{PC}$ of the signal wave into the selfconjugation regime. Namely, affecting the signal wave $G(\mathbf{r})$ by this partial operator of a $\mathrm{QH}$ results in reconstruction of its PC-replica

$$
\begin{aligned}
& \left\{2 T_{2} \Omega_{1}(\mathbf{r}) \Omega_{2}(\mathbf{r})\left(G^{*}(\mathbf{r})\right)^{2}\right\} G(\mathbf{r})= \\
& =\alpha A \exp [i(\omega t+\mathbf{k r}-\varphi)],
\end{aligned}
$$

within the complex factor $\alpha=2 T_{2} a b \exp \left[i\left(\varphi_{1}+\varphi_{2}\right)\right] \times$ $\times|G(\mathbf{r})|^{2}$.

Physically, a QH (2) is read out only with the signal wave, in absence of both components of a standing reference wave involved into the recording process. Thus, a static QH recorded into FWDM arrangement is read out into the self-conjugation mode similarly to SBS-PC. This statement can be re-formulated in terms of spatial-frequency heterodyning (non-linear mixing) of two sets of cross-gratings resulting from the interference of the signal wave with each of two components of the standing reference wave (within the frame of the secondorder hologram concept [12]), see Fig. 1. $\mathbf{K}_{\mathbf{1}}=\mathbf{k}_{\mathbf{0}}-\mathbf{k}$ and $\mathbf{K}_{2}=-\mathbf{k}_{\mathbf{0}}-\mathbf{k}$ are the vectors of interferentionally produced cross-gratings. The quadratic term of Eq. (2) describes the result of spatial-frequency heterodyning of such gratings, which partly results in appearance of the differential and total "pseudo-gratings" [13]. As a rule, the pseudo-gratings do not satisfy the Bragg rule. Just owing to this circumstance, volume nonlinearly recorded (in particularly, phase) holograms reconstruct the images that are free of nonlinear noise [13]. In our case, however, the remarkable exception to this general rule takes place. Namely, the total gratings with the grating vectors $\mathbf{F}^{(+)}=\mathbf{K}_{\mathbf{1}}+\mathbf{K}_{2} \equiv-2 \mathbf{k}$ occur to be the Bragg ones for the read out signal wave with the wavevector $\mathbf{k}$ : $\mathbf{F}^{(+)}+\mathbf{k}=-\mathbf{k}$.

Eq. (4) (the vector equivalent of Eq. (3)) is illustrated by the vector diagram Fig. 1. 


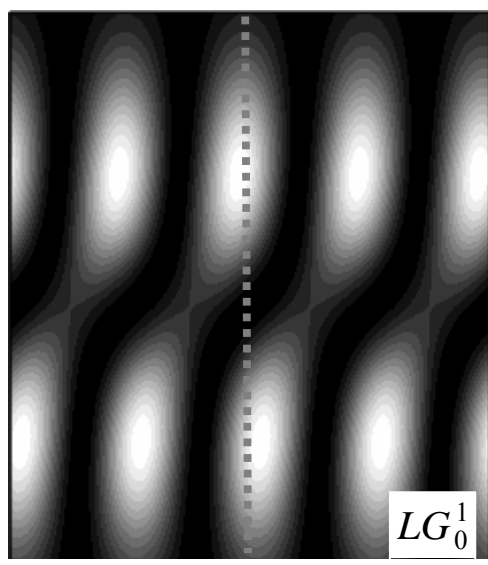

a)

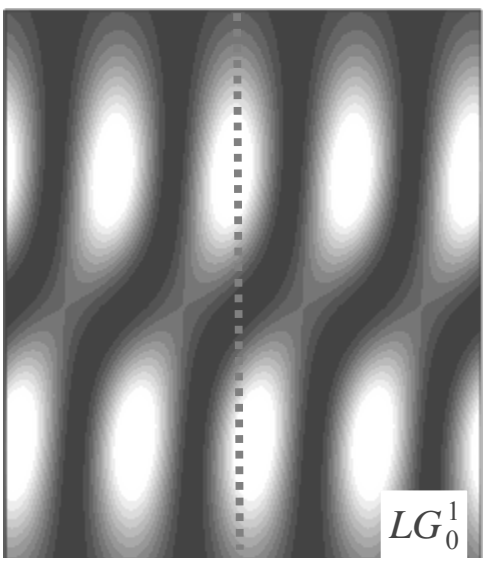

b)

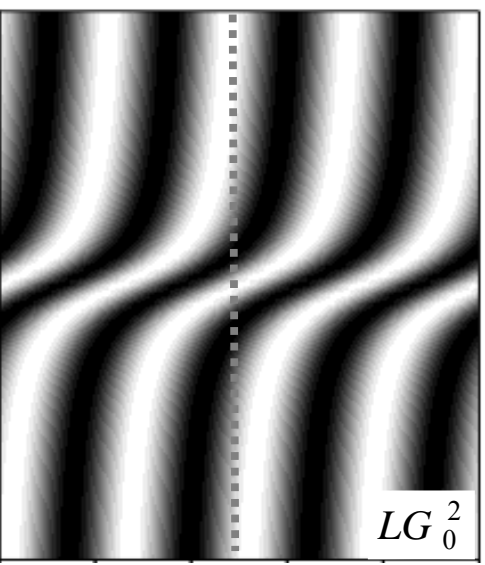

c)

Fig. 3. Simulated interferential patterns behind an opaque strip illuminated by the vortexless mode $L G_{0}^{0}$ (a) as well as doughnut vortex modes $L G_{0}^{-1}$ (b) and $L G_{0}^{-2}$ (c).

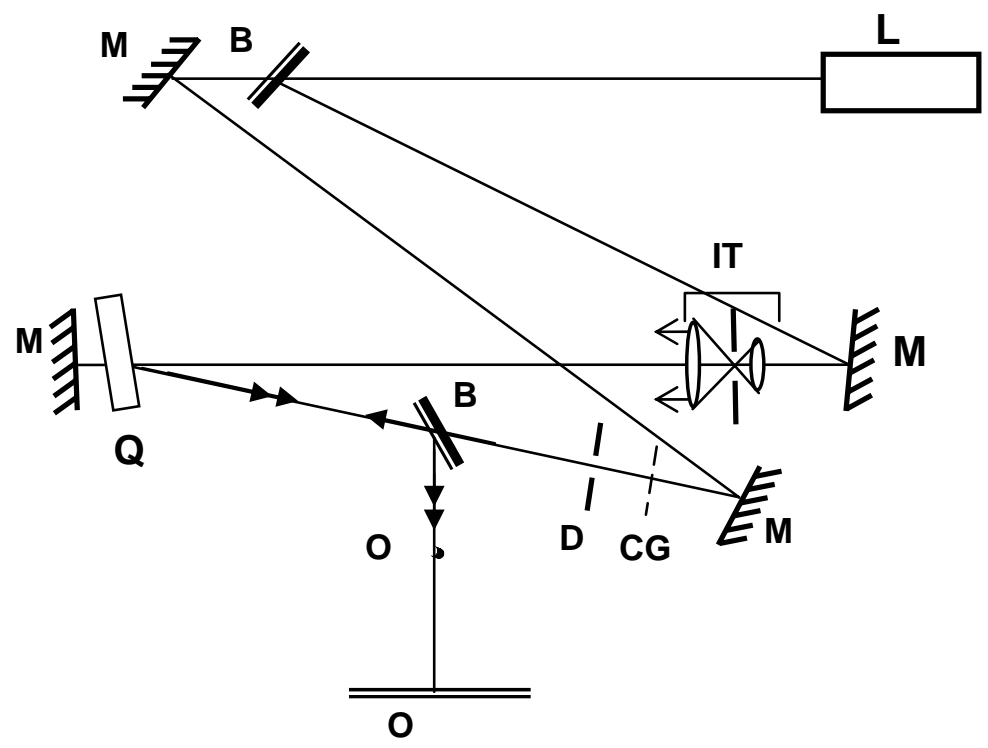

Fig. 4. Experimental arrangement for $\mathrm{PC}$ of optical vortices by a $\mathrm{QH}$ and their diffraction test: $\mathrm{L}$ - laser, $\mathrm{BS}$ - beam-splitter, $\mathrm{M}$ mirror, IT - inverse telescopic system, CGH - computer-generated hologram, D - diaphragm selecting the operating diffraction order, QH - quadric hologram - PC-mirror, OS - opaque strip, OP - observation plane.

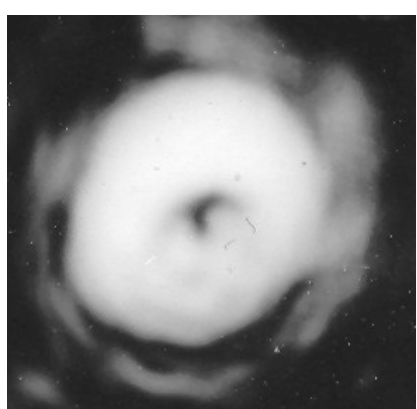

a)

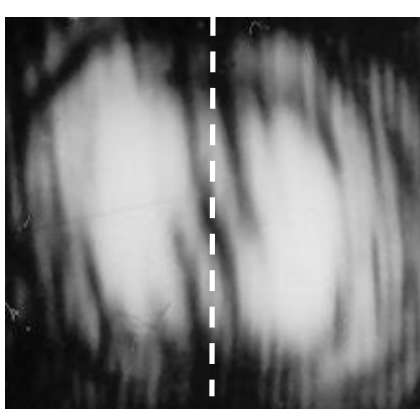

b)

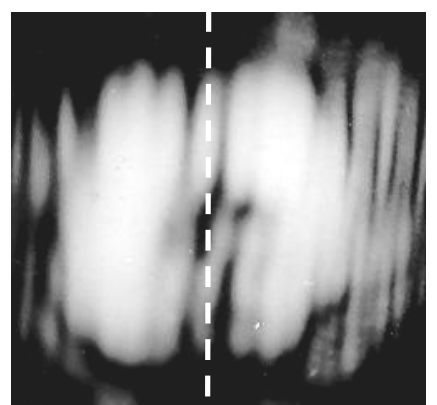

c)

Fig. 5. Laguerre-Gaussian mode $L G_{0}^{+1}$ (a), diffraction test of using an opaque strip (b), diffraction test of the self-conjugated (and mirrored) vortex (c). 


\section{Young's diagnostics of optical phase singularities}

Diagnostics of phase-conjugated vortices was performed by applying the technique based on the YoungRubinowicz model of diffraction phenomena (the edge diffraction wave model) [14-16]. This technique were recently introduced in works [17-19] and successfully used for testing the phase singularities into quasimonochromatic spatially partially coherent vortex beams $[20,21]$ and into spatially coherent polychromatic ("rainbow") optical vortices [22], as well as for diagnostics of polarization singularities into combined vortex beams [23].

The essence of diffraction diagnostics of optical vortices consists in the following. In accord with the Young-Rubinowicz model of diffraction phenomena, a diffraction pattern within the directly illuminated area behind an opaque screen is determined as the sum of the geometrical-optical wave from the primary source and the wavelets from the edges of the screen, while the pattern within the geometrical shadow is determined as the result of interference of the only edge wavelets.

Interferential patterns within a shadow of an opaque strip positioned in vortexless and vortex beams are noteworthy different. In accord with the stationary phase principle $[15,16]$, a pattern at any height $r$ from an "equator" of the beam is determined by the so-called critical points of the second kind positioned at the strip edges at the same height $r$ (see Fig. 2). When an opaque strip is illuminated by a vortexless beam, the corresponding retransmitters from the right and left edges are in-phase. As a consequence, one observes straight Young's interference fringes with peaks along the mean line of the shadow. On the contrary, when the tested beam is the $m$-charged vortex, one observes bended interference fringes with the maximum (for even $|m|$ ) or with the minimum (for odd $|m|$ ) at the equator. Generally, the phase of the Young interference fringes in the case of interest obeys the rule:

$\Delta \varphi(r, d)=m[\pi+\arctan (r / d) \mid$,

where $d$ is a halfwidth of an opaque strip. It follows from Eq. (5) that the value of interference fringe bending is determined by the modulus of the vortex topological charge, and the direction of bending is determined by the sign of the topological charge (or the direction of phase twirling). Fig. 3 illustrates the result of simulation of interferential patterns behind the opaque strip illuminated by the vortexless Laguerre-Gaussian mode and the doughnut Laguerre-Gaussian modes with topological charges $m=-1$ and $m=-2$, for $d / w_{z}=0.4\left(w_{z}\right.$ is a halfwidth of the beam at a distance $z$ from the caustic neck estimated by the level $e^{-2}$ from the maximal intensity). Dashed lines of fragments $b$ and $c$ in Fig. 3 show the shift of the interference fringes at the "poles" of the beam for one and two periods, respectively.

\section{Experiment}

The experimental arrangement for PC of vortex beams by a $\mathrm{QH}$ and for a diffraction diagnostics of the selfconjugated optical vortices is shown in Fig. 4. The beam of a He-Ne laser $(\lambda=0.63 \mu \mathrm{m}$, the power $P=40 \mathrm{~mW})$ is splitted into a signal and reference beams. Along the reference leg, the inverse telescopic system serves as the beam expander, and the mirror just behind a registration medium is used to form a plane standing reference wave. The reference leg is adjusted with the autocollimation accuracy. The singular beam is produced using an offaxis CGH $[1,5]$ computed for reconstructing the $m$ charged vortex into the $m$-th diffraction order. The operating diffraction order is selected by a diaphragm. In our experiments, we use the first and third orders of a phase CGH. Using a neutral attenuators (not shown in Fig. 3), we provide the amplitude ratio of the exposing beams $A: a: b \approx 1: 1: 1$. Nonlinear holograms are recorded on holographic photoplates PFG-03 with processing in the developer GP-2. So, we obtain phase holograms with the high-diffraction efficiency both in the first and higher diffraction orders. A distance from a CGH (the plane of the optical vortex formation) to the beam splitter decoupling the self-conjugated beam is $135 \mathrm{~mm}$. The distance from the decoupling beam splitter to the opaque screen (the plane of analysis of the selfconjugated vortex) is changed from 100 to $185 \mathrm{~mm}$. A metallic 1-mm diameter needle is used as an opaque screen. The distance from the needle to the plane of observation of the Young interference fringes is $330 \mathrm{~mm}$; for that $d / w_{z} \approx 0.77$. (Let us note that the latter appointed distance is not critical being chosen from the considerations of experimental convenience.)

The experimental results are represented in Figs 5 and 6. Fig. 5 shows the Laguerre-Gaussian mode $L G_{0}^{+1}$ and the result of diffraction testing both this mode and its self-conjugated replica. Bending the Young interference fringes in Fig. 5b indicates the clockwise twirling of a phase helicoid of the forward-propagating vortex, in agreement with the results [18, 19] and discussion of Eq. (5). On the contrary of common mirroring, which changes the direction of twirling of a phase helicoid, PC of a vortex preserves the sign of its topological charge. This difference is the noteworthy confirmation of PC properties inherent to the hologram. Note, the previous investigation of a QH-based associative memory [9-11] dealt mainly with the spatial intensity distribution in the self-conjugated associative response (to provide a true brightness tone rendering over the reconstructed image), rather than with a fine phase structure of the self-conjugated field. Here, we represent the first direct 
a)

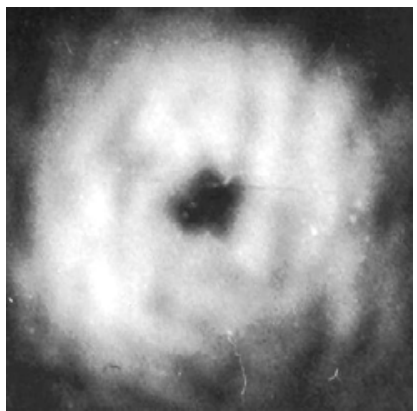

b)

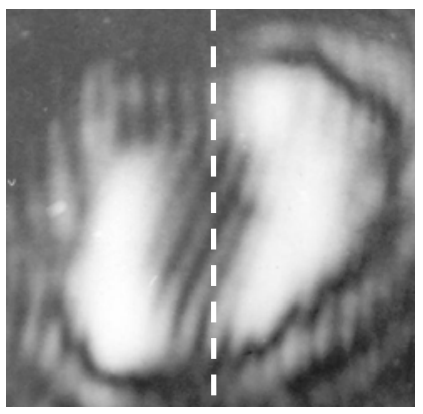

Fig. 6. Laguerre-Gaussian mode $L G_{0}^{+3}$ (a) and the diffraction test of its self-conjugated (and mirrored) replica near the PC plane (b).

confirmation of the PC properties of a static hologram nonlinearly recorded with a standing reference wave. Namely, the self-conjugated vortex must produce the Young interferential fringes (observed into the inverted coordinates!) behind an opaque strip with identical bending as that observed in the forward-propagating beam observed in initial coordinates, while the spatial (three-dimensional) configuration of a phase helicoids is preserved under alternating the direction of the beam propagation. Nevertheless, in Fig. 5c, one observes bending the interference fringes that is opposite to that shown in Fig. 5b. Moreover, the experiment shows that the common mirroring of an optical vortex results in the same change of the topological charge. This circumstance has the following explanation [4]. Inversion of a phase twirling observed in our PC experiment is caused by the mirroring from the decoupling beam splitter guided the self-conjugated vortex into the observation plane. It means that the selfconjugated optical vortex between a $\mathrm{QH}$ and a beam splitter possesses the twirling of the phase helicoid intrinsic to the true PC. The result shown in Fig. 5c is observed both in the plane of PC and at arbitrary distances from this plane. Thus, the singly charged selfconjugated optical vortex is topologically stable.

At last, Fig. 6 shows PC of the multiply charged (with $|m|>1$ ) optical vortex. Fig. 6a demonstrates the Laguerre-Gaussian mode $L G_{0}^{+3}$ reconstructed into the third diffraction order of the same $\mathrm{CGH}$, which was used for obtaining the results shown in Fig. 5. Selfconjugated replica of this beam undergone diffraction testing (for the distance from the decoupling beamsplitter to the opaque strip $135 \mathrm{~mm}$ ) is shown in Fig. 6b. The dashed line indicates increased bending on interference fringes in accord with Eq. (5).

In contrast to singly charged optical vortices, the beams with $|m|>1$ generate the self-conjugated replicas through diffraction of the initial beam at a $\mathrm{QH}$ only in the limited region near the PC plane. As a matter of fact, the multiply charged vortices are topologically instable [1]. The propagating multiply charged vortex decays into elementary (singly charged) vortices of the same sign, which are repulsed similarly to electrical charges [4]. That is why, observation of the multiply charged vortices into random fields, such as developed speckle fields, is hampered. In the case studied in this paper, we operate with optical vortices artificially reconstructed from a $\mathrm{CGH}$, which provides self-conjugation of the multiply charged phase helicoids, though in the limited region. The diffraction technique for diagnostics of singular beams used here provides a possibility to observe decaying the multiply charged vortices into the elementary ones under their propagation. So, when an opaque strip moves from the PC plane, the single set of Young's interferential fringes with $m$-fold increased bending decays into the set of $m$ sets corresponding to the singly charged vortices with the same direction on fringe bending.

\section{Conclusions}

Thus, in this paper, we represent a PC of vortex optical beams using a hologram nonlinearly recorded with a standing reference wave. PC is provided by the combination (summation) gratings associated with the quadratic component of an amplitude response of such a hologram. The main practical pre-request of holographic recording nonlinearity, namely, signal-to-reference amplitude ratio close to unity, providing high visibility of the resulting interferential pattern (deep spatial inhomogeneity of the exposure field) ensures the possibility for high-efficient $\mathrm{PC}$ of a vortex beam into self-conjugation mode using a static $\mathrm{QH}$ recorded with a standing reference wave.

To test phase singularities into phase-conjugated optical vortices, for the first time, we have applied the diffraction technique by which the PC properties of a $\mathrm{QH}$ have been confirmed experimentally. It has been shown that the spatial configuration of a phase helocoid of the self-conjugated vortex differs from such configuration resulting from a common vortex mirroring.

At last, the possibility of self-reconstruction of the multiply charged vortices at the PC plane has been demonstrated, for example, the Laguerre-Gaussian mode with $|m|=3$. Such reconstruction has been confirmed 
using the diffraction diagnostic technique on the correspondingly increased bending the Young interference fringes inside the shadow of an opaque strip.

In some specified sense, the represented here effect of PC of optical vortices corresponds to the earlier studied vector PC of circularly polarized vortexless beams using a static hologram recorded nonlinearly with two oppositely directed orthogonally linearly polarized reference waves [8]. In the case considered here, transformation of the spatial structure of a phase helicoids is associated with that of the orbital angular momentum of a vortex beam, while in the case of vector PC one considers the transformation of the spin moment intrinsic to a circularly polarized signal beam. Nevertheless, general regularities of transformation of such beams following from the PC properties of a $\mathrm{QH}$ are identical. So, in both cases the result of observation depends on the choice of coordinates (on direction of beam propagation), and the interpretation of the observed results presumes accounting the additional transformation of the beam structure due to the reflection of the self-conjugated beam at the beam splitter decoupling this beam in the observation plane.

\section{References}

1. M.S. Soskin, M.V. Vasnetsov, Singular Optics // Progress in Optics, ed. by E. Wolf, Elsevier, Amsterdam, 42, p. 219-276 (2001).

2. P. Yeh, Introduction to Photorefractive Nonlinear Optics, Wiley, New York (1993).

3. I.G. Marienko, M.S. Soskin, M.V. Vasnetsov, Phase conjugation of wavefronts containing phase singularities // Proc. SPIE 3487, p. 39-41 (1998).

4. I.G. Marienko, M.S. Soskin, M.V. Vasnetsov, Phase reversal of laser beams carrying optical vortices // Asian J. Phys. 7, p. 495-501 (1998).

5. V.Yu. Bazhenov, M.V. Vanetsov, M.S. Soskin, Laser beams with screw dislocations of wave front // Sov. JETP Lett. 52, p. 429-431 (1990).

6. N.R. Baranova, A.V. Mamayev, N.F. Pilitetskii, V.V. Shkunov, B.Ya. Zel'dowich, Wavefront dislocations: topological limitations for adaptive systems with phase conjugation // J. Opt. Soc. Amer. 73, p. 525-528 (1983).

7. G.V. Bogatyryova, M.S. Soskin, Detection and metrology of optical vortex helical wave fronts // Semiconductor Physics, Quantum Electronics \& Optoelectronics 6, p. 254-258 (2003).

8. P.V. Polyanskii, On polarization phase conjugation of a wave front by a quadric hologram // Opt. Spectr. 83, p. 324-326 (1997).

9. P.V. Polyanskii, Associative memory using a quadric hologram-based phase-conjugate mirror // Ibid. 84, p. 300-305 (1998).

10. P.V. Polyanskii, On the frontiers of the phaseconjugating effect using a nonlinear hologram // Ibid. 86, p. 288-292 (1999).

11. P.V. Polyanskii, G.V. Bogatyryova, Contoured phase-conjugated response of a quadric hologram // Opt. Appl. 29, p. 583-600 (1999).

12. H.J. Caulfield, Holography and information: getting something for nothing with light // Proc. Intern. Confer. "From Galileo's "occialino" to optoelectronics", World Sc. Publishing, New York, p. 34-49 (1993).

13. J. Upatnieks, C. Leonard, Efficiency and image contrast of dielectric holograms // J. Opt. Soc. Amer. 60, p. 297-305 (1970).

14. Th. Young, On the theory of light and colours // Philos. Trans. Roy. Soc. London 20, p. 26 (1802).

15. A. Sommerfeld, Optics, Academic Press, New York (1954).

16. K. Miyamoto, E. Wolf, Generalization of the YoungRubinowicz theory of the boundary diffraction wave, Parts I and II // Journ. Opt. Soc. Amer. 52, p. 615637 (1962).

17. P.V. Polyanskii, Some current views on singular optics // Proc. SPIE 5477, p. 31-40 (2003).

18. Ch.V. Felde, Diffraction testing of singular beams // Ibid. p. 67-76 (2003).

19. G.V. Bogatyryova, Ch.V. Felde, P.V. Polyanskii, Referenceless testing of vortex optical beams // Opt. Appl. 33, p. 695-708 (2003).

20. G.V. Bogatyryova, Ch.V. Felde, P.V. Polyanskii, S.A. Ponomarenko, M.S. Soskin, E. Wolf, Partially coherent vortex beams with a separable phase // Opt. Lett. 28, p. 878-880 (2003).

21.Ch.V.Felde, Young's diagnostics of phase singularities of the spatial coherence function at partially coherent singular beams // Ukr. J. Phys. 49, p. 473-480 (2004).

22. O.O. Arkhelyuk, P.V. Polyanskii, A.A. Ivanovskii, M.S. Soskin, Creation and diagnostics of stable rainbow optical vortices // Opt. Appl. 34, p. 419-426 (2004).

23. G.V. Bogatyryova, Ch.V. Felde, P.V. Polyanskii, M.S. Soskin, Nongeneric polarization singularities into combined vortex beams // Opt. Spectr. 97, p. $833-840$ (2004). 